\title{
Family and the Risky Behaviors of High School Students
}

\author{
Aliakbar Haghdoost ${ }^{1}$; Faroukh Abazari ${ }^{2,} ;$ Abbas Abbaszadeh $^{3}$; Eshagh Dortaj Rabori $^{4}$ \\ ${ }_{1}^{1}$ Research Center for Modeling in Health, Kerman University of Medical Sciences, Kerman, IR Iran \\ 2 Department of Community Health, Nursing and Midwifery Kerman, School of Razi, Kerman University of Medical Sciences, Kerman, IR Iran \\ 3 Department of Medical Surgical Nursing, Shahid Beheshti University of Medical Sciences, Tehran, IR Iran \\ 4 Department of Epidemiology, School of Health Sciences, Kerman University of Medical Sciences, Kerman, IR Iran \\ ${ }^{*}$ Corresponding Author: Faroukh Abazari, Department of Community Health, Nursing and Midwifery Kerman, School of Razi, Kerman University of Medical Sciences, Kerman, IR \\ Iran. Tel: +98-3413205643, Fax:+98-3413205642, E-mail: f_abazari@KMU.ac.ir \\ Received: November 2, 2013; Revised: April 17, 2014; Accepted: July 13, 2014
}

\begin{abstract}
Background: Family plays an important role in helping adolescent acquiring skills or strengthening their characters.
Objectives: We aimed to evaluate the influences of family factors, risky and protective, on adolescent health-risk behavior(HRB).

Patients and Methods: In this cross-sectional study, students of high schools in Kerman, Iran at all levels participated, during November 2011 till December 2011. The research sample included 1024 students (588 females and 436 males) aged 15 to 19 years. A CTC (Communities That Care Youth Survey) questionnaire was designed in order to collect the profile of the students' risky behaviors. Stratified cluster sampling method was used to collect the data.

Results: Using logistic regression, 7 variables enrolled; 4 of them were risk factors and 3 were protective factors. The risk factors were age, (linear effect, $\mathrm{ORa}=1.20, \mathrm{P}=0.001$ ), boys versus girls $(\mathrm{ORa}=2.33, \mathrm{P}=0.001)$, family history of antisocial behavior $(\mathrm{ORa}=2.29, \mathrm{P}=0.001)$, and parental attitudes favorable toward antisocial behavior $(\mathrm{ORa}=1.72, \mathrm{P}=0.03)$. And, protective factors were family religiosity $(\mathrm{ORa}=0.65, \mathrm{P}=$ 0.001 ), father education (linear effect, $\mathrm{ORa}=0.48, \mathrm{P}=0.001$ ), and family attachment (ORa $=0.78, \mathrm{P}=0.001$ ).

Conclusions: Our findings showed that family has a very significant role in protecting students against risky behaviors. The education level of the father, family religiosity, and attachment were the most important factors.
\end{abstract}

Keywords:Parents; Risk; Health; Behaviors; Adolescent

\section{Background}

Adolescents are valuable resources in human societies who encounter risk factors because of their age and evolutionary features (1). Sometimes, adolescent risk factors may last until adulthood and will become harmful for them and others (2). Risky and protective factors can affect children at different stages of their lives. At each stage, risky events occur that can be prevented through intervention measures. Early-childhood risks like aggressive behavior, can be changed or prevented by family, school, and community. These interventions focus on helping children develop appropriate and positive behaviors. If negative behaviors are not addressed properly, they can lead to worse situations such as academic failure and social difficulties, which put children at further risk like drug abuse (3). When a child enters adolescence, his or her family communications change drastically and attain a new form (4). Adolescent's ongoing attempts to achieve autonomy can result in increased parent-child conflicts at the beginning of this stage and negative feelings during this period.

These conflicts mainly happen because of different expectations of suitable behavior from both parents and children, as well as conflicting understanding of the responsibility, independence, and duties (5). The family is the fundamental factor in supporting adolescents emo- tionally, economically, and providing them an identity and feeling of belonging (6). Any kind of positive or negative change in the family has a direct effect on the larger human society. Family stability or instability directly affects the society. Thus, in societies where family values are unstable, moral values are considered irrelevant. Although adolescents are susceptible to risky behaviors, there are factors such as religious activities, good relationship with parents, and parental support that might buffer against the adolescent's tendency towards highrisk behaviors $(7,8)$.

The concept of health-risk behavior can be defined as; any activity undertaken by people with a frequency or intensity that increases their risk of disease or injury such as substance abuse, risky driving, violence or suicidal tendencies, and antisocial behavior $(9,10)$. There is evidence that health-risk behaviors tend to cluster together with similar risk factors, underlying a lot of risk behaviors (11, 12). Inquiries into risk behaviors and protective factors among adolescents are prominent in the social, behavioral, and health sciences, and include study of particular risk factors (13). The significant role of family and its environment on adolescents' tendency towards high-risk behaviors, and the increasing rate of this problem among

Copyright (C) 2014, Iranian Red Crescent Medical Journal; Published by Kowsar. This is an open-access article distributed under the terms of the Creative Commons Attribution-NonCommercial 4.0 International License (http://creativecommons.org/licenses/by-nc/4.0/) which permits copy and redistribute the material just in noncommercial usages, provided the original work is properly cited. 
Iranian adolescents has led many scholars to focus on this important social issue $(14,15)$.

Iran is an Islamic country, respecting family and is currently undergoing a transition towards a modern society. It has a special cultural condition, which emphasizes Islamic values. Because, Iran is in transition from a traditional society to a modern and industrial one, damages to family roles and relations are important problems (16). Moreover, in developing countries, the key role of family in educating adolescents and its effect on juvenile delinquency seems more important than that in western countries. Severe behavioral controls, which are imposed on adolescents by various organizations, also make the range and conditions of adolescent behaviors so different from western countries.

On the other hand, cultural issues of every society must be considered while speaking about risky behaviors. Values and norms of every society are effective in the pattern of these behaviors (17). Olds' reviews revealed that social norms are the strongest factor for participating in risky behaviors (18). Although there are some studies in this field in Iran, most published papers explored only the frequency of risk behaviors among Iranian students. This paper, however, presents comprehensively the results of an analytical study which explores the relationship between family factors and the profile of student's behavior.

\section{Objectives}

We aimed to evaluate family and its effects on the risky behaviors among Iranian high school students in SouthEast of Iran.

\section{Materials and Methods}

\subsection{Participants}

The present research is a cross-sectional study, carried out among high school students in one of the main cities in the southeast of Iran (Kerman), with a population of more than 650 000, from November to December 2011. The research sample included 1024 students aged 15-19 years, representing all levels of the high school (first to third grades and pre-university). Eligible schools included any high school with students in their first to third grades and pre-university in Kerman. Students, who were transferring permanently from another city to a Kerman high school during the study period, were not included in the research. In addition, high schools without all grades were excluded from our sample. In our sample, around $90 \%$ of schools were included. By receiving permission of the Education Department's Counseling Center in Kerman, we selected our subjects using a stratified cluster sampling technique.

First, we classified high schools based on their gender, location (north, west, east, and south), and type, either governmental or private. Then, in proportion to size, we selected schools randomly, while students were selected from different grades within their classes. All participants were informed about the goals of the survey and received the guidelines and instructions to fill out the questionnaire. Participants signed written informed consent and then completed the questionnaires anonymously.

\subsection{Instrument}

We used "Communities That Care Youth Survey" to assess a broad set of risk and protective factors across the domains of family, school, community, peer, and individual as well as health-risk behavior outcomes. This questionnaire was prepared by Hawkins and Catalano (19, 20). In this study, we only used a part of this questionnaire, which measured family domain. The questionnaire consisted of an index of problem behaviors, including 14 items (which measured their frequency during the previous months or year) such as smoking, aggression, fighting, weapons carrying, and suspension from school. An index of protective factors assessing by family rewards for prosocial involvement included 3 components. Assessment of family attachment had 3 components. Family religiosity was assessed by 4 components and family opportunities for prosocial involvement by 4 components. Risk factors consisted of poor family management with 8 components, family conflict with 3 components, family history of antisocial behavior with 7 components and parental attitudes favorable toward antisocial behavior with 4 components.

Validity of the questionnaire was ensured through 3 stages, including scale translation, face validity, and content validity. Regarding scale translation, we used the procedure of forward-backward translation. Then, it was revised by four health education and panel members. They were asked to review each item and evaluate the appropriateness of the translated items for face validity, in other words, to be understandable by the research target. The content validity of CTC questionnaire was investigated both quantitatively and qualitatively by the same experts. We asked the experts to evaluate the quality and quantity of each item of CTC questionnaire. Necessity, relevancy, simplicity, and clarity of each item were assessed using Likert 5-point type scale. An open question was also asked to elicit the opinions of the experts concerning each item. Content validity index (CVI) was computed on the basis of the simplicity, clarification, and relevancy of each item. ACVI score of higher than 0.75 was considered reasonable. Content validity ratio (CVR) scores were calculated based on the necessity of each item. A CVR score of equal to or higher than 0.59 was envisaged a good content validity by 10 experts. The mean of CVI and CVR was 0.87 and 0.78 , respectively, signifying a good content validity for CTC questioner.

Using the test-retest technique, 40 students (20 girls, 20 boys) responded twice with a gap of 10 days between the two assessments. The consistency between their scores was computed by the Pearson correlation coefficient of 0.75. Additionally, we computed the Cronbach $\alpha$ value for all participants after the data collection, which was at 
0.78. The questions of each risky behavior style were measured by 5 items that were rated on a 5-point Likert scale ranging from never to more than 10 times in the last 30 days or last year. The presence of floor and ceiling effects may influence the reliability, validity, and responsiveness of an instrument. In order to determine floor and ceiling effects, we calculated the percentage of student with very low and very high scores. The rates of floor effect and ceiling effect were calculated for each scale in all questionnaires and were considered suitable when it was below $15 \%$, because there was no consensus on how to define floor and ceiling effects mathematically.

\subsection{Procedure}

Students in grades first to third and pre-university who were enrolled in high schools in Kerman were targeted during the study period. We collected consent form from the students and their parents separately. Students whose parents and themselves provided written consent to participate were identified by the school manager. Having checked with the schools, students were approached in their classes, but they answered the questions in a private environment and their responses were collected without any identifiers. In order to assess the associations between HRB and family factors, we estimated the sample size by comparing two mean formulas. In this calculation and were set at $5 \%$ and $10 \%$, and the minimum effect size of 0.5 of the standard deviation, and design effect of 1.5. Based on these assumptions, the estimated sample size was 1050.

\subsection{Data Analyses}

The data was computerized and analyzed using the statistical package of social sciences (SPSS) version 18, and before data entry, all completed questionnaires were evaluated by the main investigator. Then, the distribution of the responses was assessed and the main variables were described. In the next step, risky behaviors were divided into two groups: low-risk if the subject had smoking, aggression behaviors less than three times per month and weapons carrying, fighting, suspension from school less than three times per year, and high-risk groups if the subject had exposure to the above-mentioned items more than three times.

In this analysis, the main dependent variables were age (in year) gender, grade (first to third grades and pre- university), risk and protective factors in the family (in 8 subscales, each one had a score between 0 and 4). Using logistic regression, crude and adjusted ORs between having risky behaviors and other independent variables (sociodemographic variables, risk and protective factors in the family) were computed. In the final multivariate model, only the significant variables in crude models were entered.

\section{Results}

A total of 1024 students between 15 and 19 years of age (57.4\% females, mean of age $=16.4, \mathrm{SD}= \pm 1.5$ year) completed the questionnaires. The percentage (number) of students in grades first to third and pre-university were 28\% (287); 26.6\%(272); 26.4\% (270); and 19\% (195), respectively (Table 1) The number of students stated that they didn't practice any risky behaviors was 443 (41.7\%). Conversely 307 (52.3\%) girls and 136 (31.2\%) boys and 13 (2.2\%) of girls and 29 (6.7\%) of boys experienced more than 6 instances of HRB (Table 2). A significant positive association was found between age and the frequency of HRB (crude add ratio $(\mathrm{ORc})=1.23, \mathrm{P}=0.01$, adjusted odds ratio $(\mathrm{ORa})=1.20, \mathrm{P}=$ 0.001 . The results of the logistic regression model showed that boys had more HRB ( ORc $=2.40, \mathrm{P}=0.001$; ORa $=2.33$, $\mathrm{P}=0.001)$. Although the association between grade levels and $\mathrm{HRB}$ was significant in the univariate analysis (ORc = $1.16, \mathrm{P}=0.001)$; it was not significant in the multivariate model $(\mathrm{ORa}=0.90, \mathrm{P}=0.42)$. The number of sibling in family ( $\mathrm{ORc}=1.28, \mathrm{P}=0.001)$ had a significant positive association with $\mathrm{HRB}$, but its association was not significant in the multivariate model $(\mathrm{ORa}=0.98, \mathrm{P}=0.78$ ).

Table 1. Demographic Characteristics of Students in Kerman High Schools ${ }^{a}$

\begin{tabular}{lc}
\hline Variables & Results, No. (\%) \\
\hline Gender & \\
\hline Female & $588(57.4)$ \\
\hline Male & $436(42.6)$ \\
Grade differences & $287(28)$ \\
\hline Grade 1 & $272(26.6)$ \\
Grade 2 & $270(26.4)$ \\
Grade 3 & $195(19)$ \\
\hline Grade 4 & \\
\hline${ }^{a}$ Data are presented as No.(\%).
\end{tabular}

\begin{tabular}{|c|c|c|c|c|c|c|c|c|}
\hline & о & 1 & 2 & 3 & 4 & 5 & +6 & \\
\hline Females & $307(52.3)$ & $146(24.8)$ & $62(10.6)$ & $32(5.4)$ & $17(2.9)$ & $11(1.9)$ & $13(2.2)$ & $588(100)$ \\
\hline \multicolumn{9}{|l|}{ Age, $y$} \\
\hline 15 & $102(48.6)$ & $59(28.1)$ & $23(11.0)$ & $13(6.2)$ & $7(3.3)$ & $2(1.0)$ & $4(1.9)$ & $210(100)$ \\
\hline 16 & $144(47.5)$ & $72(23.8)$ & $33(10.9)$ & $28(9.2)$ & $7(2.3)$ & $6(2.0)$ & $13(4.3)$ & $303(100)$ \\
\hline 17 & $138(42.5)$ & $85(26.2)$ & $40(12.3)$ & $24(7.4)$ & $11(3.4)$ & $15(4.6)$ & $12(3.7)$ & $325(100)$ \\
\hline $18+19$ & $59(31.7)$ & $50(26.9)$ & $25(13.4)$ & $15(8.1)$ & $14(7.5)$ & $10(5.4)$ & $13(7.0)$ & $186(100)$ \\
\hline Total & $443(43.3)$ & $266(26.0)$ & $121(11.8)$ & $80(7.8)$ & $39(3.8)$ & $33(3.2)$ & $42(4.1)$ & $1024(100)$ \\
\hline
\end{tabular}

\footnotetext{
${ }^{\mathrm{a}}$ Data are presented as No.(\%).
} 
Table 3. Logistic Regression Model, the Association Between the Presence of Health Risk Behavior and Family Variables Among Participating Students in Kerman High Schools

\begin{tabular}{|c|c|c|c|c|}
\hline Variable & Crude Odd Ratio & P Value & Adjusted Odd Ratio & P Value \\
\hline Linear effect of age, $y$ & $1.23(1.10-1.38)$ & 0.001 & $1.20(1.03-1.36)$ & 0.001 \\
\hline \multicolumn{5}{|l|}{ Gender } \\
\hline Female & 1 & & 1 & \\
\hline Male & $2.40(1.86-3.12)$ & 0.001 & $2.33(1.70-3.17)$ & 0.001 \\
\hline \multicolumn{5}{|l|}{ Grade level } \\
\hline Grade 1 & 1 & & 1 & \\
\hline Grade 2, 3, 4 & $1.16(1.03-1.30)$ & 0.001 & $0.90(0.61-1.07)$ & 0.42 \\
\hline \multicolumn{5}{|l|}{$\begin{array}{l}\text { Mother education (Linear } \\
\text { effect) }\end{array}$} \\
\hline $\begin{array}{l}\text { Primary, secondary and high } \\
\text { school }\end{array}$ & 1 & 0.001 & 1 & 0.58 \\
\hline University & $1.29(1.06-1.32)$ & & $1.02(1.10-1.59)$ & \\
\hline \multicolumn{5}{|l|}{$\begin{array}{l}\text { Father education (Linear } \\
\text { effect) }\end{array}$} \\
\hline $\begin{array}{l}\text { Primary, secondary and high } \\
\text { school }\end{array}$ & 1 & 0.001 & 1 & 0.001 \\
\hline University & $0.60(0.41-0.88)$ & & $0.48(0.28-083)$ & \\
\hline Number of siblings in family & $1.28(0.93-1.29)$ & 0.001 & $0.98(0.91-1.08)$ & 0.78 \\
\hline $\begin{array}{l}\text { Family rewards for prosocial } \\
\text { involvement }\end{array}$ & $0.72(0.61-076)$ & 0.001 & $0.92(0.74-1.17)$ & 0.51 \\
\hline Family attachment & $0.66(0.58-0.73)$ & 0.001 & $0.78(0.70-1.01)$ & 0.001 \\
\hline Family religiosity & $0.57,(0.47-0.67)$ & 0.001 & $0.65(0.54-0.86)$ & 0.001 \\
\hline $\begin{array}{l}\text { Family opportunities for } \\
\text { Prosocial involvement }\end{array}$ & $0.60,(0.54-0.71)$ & 0.001 & $0.94(0.78-1.16)$ & 0.56 \\
\hline Poor family management & $1.96(1.57-2.44)$ & 0.01 & $1.23(0.91-1.33)$ & 0.12 \\
\hline Family conflict & $1.15(1-1.33)$ & 0.001 & $0.98(0.81-1.16)$ & 0.83 \\
\hline $\begin{array}{l}\text { Family history of antisocial } \\
\text { behavior }\end{array}$ & $3.11(2.38-4.07)$ & 0.04 & $2.29(1.64-3.39)$ & 0.001 \\
\hline $\begin{array}{l}\text { Parental attitudes favorable } \\
\text { toward antisocial behavior }\end{array}$ & $2.54(1.85-3.48)$ & 0.01 & $1.72(1.10-2.68)$ & 0.03 \\
\hline
\end{tabular}

Mothers' education levels had a significant positive association only in the univariate analysis (ORc $=1.29, \mathrm{P}=$ $0.001 ;$ ORa $=1.02, \mathrm{P}=0.58)$. On the other hand, fathers' education levels showed a negative association as a predictor of HRB in both models $(\mathrm{ORc}=0.60, \mathrm{P}=0.01$; ORa $=0.48, \mathrm{P}=0.001)$. Risk factors related to "the family history of antisocial behavior" had a very strong positive association with $\mathrm{HRB}$ in both models (ORc $=0.3 .11, \mathrm{P}=$ $0.04 ; \mathrm{ORa}=2.29, \mathrm{P}=0.001)$. Whereas "poor family management" showed a significant association with HRB only in the univariate model $(\mathrm{ORc}=1.96, \mathrm{P}=0.01$; ORa $=1.23$, $\mathrm{P}=0.12$ ), "parental attitudes favorable toward antisocial behavior" had a positive association with HRB in both the univariate and multivariate models $(\mathrm{ORc}=3.35, \mathrm{P}=0.001$; $\mathrm{ORa}=1.27, \mathrm{P}=0.003$. Also, "family conflict" was another variable, which had statistically significant positive asso- ciation with $\mathrm{HRB}$ in the univariate analysis $(\mathrm{ORc}=1.15, \mathrm{P}=$ 0.001). However, in the multivariate, the association was absent $(\mathrm{ORa}=0.98, \mathrm{P}=0.83)$.

Protective factors related to "family rewards for prosocial involvement" $(\mathrm{ORc}=0.72, \mathrm{P}=0.01 ; \mathrm{ORa}=0.92, \mathrm{P}=0.51)$ and "family opportunities for prosocial involvement" (ORc $=0.3 .11, \mathrm{P}=0.004 ; \mathrm{ORa}=2.29, \mathrm{P}=0.001)$, showed $\mathrm{a}$ significant negative association with HRB only in the univariate but, in the multivariate, the association in both variables were removed. In the final multivariate logistic regression, 4 variables, including age, $(\mathrm{ORa}=1.20, \mathrm{P}=$ 0.001 ), males versus females ( $\mathrm{ORa}=2.33, \mathrm{P}=0.001)$, family history of antisocial behavior $(\mathrm{ORa}=2.29, \mathrm{P}=0.001)$, and parental attitudes favorable toward antisocial behavior $(\mathrm{ORa}=1.72, \mathrm{P}=0.03)$ were risk factors for adolescents with HRB in both models. Additionally, family religiosity (ORa 
$=0.65, \mathrm{P}=0.001)$, fathers' education levels $(\mathrm{ORa}=0.48, \mathrm{P}=$ $0.001)$ and family attachment $(\mathrm{ORa}=0.78, \mathrm{P}=0.001)$ were protective factors (Table 3 ).

\section{Discussion}

The presented results provide a broad picture of the effect of family risk and protective factors on adolescents' health-risk behaviors. We found that family attachment, father education and family religiosity were protective factors. On the other hand, boys versus girls, age, family history of risky behaviors, and parental attitudes favor toward antisocial behavior, which could result in increased risky behaviors. In recent decades, dealing with the population of adolescents has become an international problem, and this problem is important in Iran. According to the traditional system in Iran, the family plays an important role in training and guiding adolescents. The presence of various competitive institutions like schools, peers, the Internet, and satellite networks, which have deep potential differences in terms of values and ideals has changed the dynamics of the family and challenged family performance.

In studying the reasons of risk factors in families, it is better to pay attention to a combination of factors and relationships and to take an effective step to prevent and treat them. Results of the present study have revealed that when adolescents grow older, they get more involved in risky behaviors. Other studies showed similar results $(21,22)$. This variable can be considered a suitable indicator of adolescent high-risk behaviors. Therefore, initial preventive programs must begin in preadolescent ages in the form of informative and warning programs and must focus more on adolescents who are at higher risks. Another individual risk factor is the role of gender in high-risk behaviors; i.e. boys are at higher risk than girls. It can be attributed to cultural features namely, cultural and educational conditions limit girls and allow boys to have more freedom. Studies have taken sex differences into account and mentioned that different cultures treat girls and boys differently, which subsequently affect their socialization and various behaviors. Studies carried out by Huebner et al. and Kapungu et al. revealed similar results $(23,24)$.

One unique contribution of the existing study was to obtain the most important risk factor which was the predictor of the adolescents' health-risk behaviors in family, i.e. a family history of risky behavior. Adolescent in the families which excuse them for breaking the law are more likely to develop problems with risky behavior. Families whose parents engage in risky behavior inside or outside the home are at greater risks for exhibiting risky behavior. Adolescents whose parents practice drug abuse have higher tendencies towards risky behaviors because they watch their parents' behavior every day and attempt to (under the influence of observational learning) select them as their models in life and act accordingly. In addition, similar studies support the results obtained in this research $(20,25)$. Parental attitudes favorable toward antisocial behavior were another risk factor. Parental attitudes do appear to be influential in their own right; for example, children whose parents behave aggressively or violently at home are more likely to become aggressive and violent adolescents $(26,27)$. However, the independent significance of this risk factor may be most relevant to drug use. A number of US studies have linked 'parental modeling' with favorable attitudes towards the use of alcohol, tobacco, and illegal drugs at home to the chances of children becoming users and abusers (20).

One of the most protective elements in the family was attachment and intimacy between family members, especially parents. It is shown that this factor has a significant effect on health-risk behaviors. In his research, Wisner (2004) concluded that poor attachment between family members, lack of parental empathy, and absence of parents at home are predictors of high-risk behaviors in children (28). Conversely, warm and intimate relationship between parents and children are the basis of emotional security in adolescents and result in strong bonds between parents and adolescents; this conformity causes improved self-esteem in adolescents, makes them spend most of their free times with their families and thus reduces high-risk behaviors (29-31).

The second protective factor was the role of religious beliefs and religious practices within the family. One of the most important factors in reducing high-risk behaviors is religious beliefs and religious practices as well as encouraging adolescents to practice religion. Religious beliefs play an important role in providing health, especially mental health. Risky behaviors are strongly influenced by religious values and beliefs; for example, it was shown in a research on 299 American adolescents that religious constraints and a sense of belonging to Muslim heritage prevented adolescents from drinking alcohol $(27,32)$. It was shown that religious beliefs prevented high-risk behaviors (33).

Surprisingly, we found a positive association between the level of mother's education and risky behavior. This type of association was also reported by another study in Iran (34). However, most studies in the world showed comparable negative associations between mothers' education levels and risky behaviors. Besides, higher education is associated with higher probability of having a job and being busy. Working mothers spend less time for emotional support, continuous supervision, encouraging and helping with school activities, and this might result in developing riskier behaviors among their children (35). Similar to other findings, our results showed that family has a key role in shaping students' behavior in Iran. A warm family with strong support, and religious practices would have a very significant role in training students. However, we should note that well educated mothers might have less impact on the risky behaviors of their children. This is most probably due to the lack of time these mothers spend with their children. 


\subsection{Limitation}

This study could not clearly determine which behavioral factor would result in other behaviors. Moreover, these findings were obtained only from students who attended the school, and thus school dropouts, students who had failed academically, those who could not enter high school and those who studied at night schools were not included. Additionally, because of the sensitivity of some subjects like smoking, students might underreport their behaviors, although by using different techniques, we attempted to convince students to response with minimum barriers.

\subsection{Human Subjects Approval Statement}

Based on the proposal of the study, the Medical Research Ethics Committee of the University of Kerman gave an approval to the researcher to conduct the survey among high school students in Kerman, and informed consents submitted to Medical Science University of Kerman. In this regard, two different written consent forms were taken: the first one involved the permission to do the study; the second one from Ministry of Education for participation in the study. After we have identified the classrooms in a school, enough parental permission forms were delivered to the principal for each selected student. The code and date of ethical approval were K/89/70-2011. We certify that there is no conflict of interests with any financial organization regarding the material discussed in the manuscript.

\section{Acknowledgements}

This article is a part of PhD thesis supported by Kerman University of Medical Sciences. We also wish to thank all the students, teachers, and principals of Kerman high schools for their valuable collaboration with this study.

\section{Authors' Contributions}

Abbas Abbaszadeh and Eshagh Dortaj Rabori contributed to the development of the protocol, and the original idea. Faroukh Abazari developed the original idea and the protocol, abstracted the data, and prepared the manuscript. Dr. Aliakbar Haghdoost analyzed the data, wrote the manuscript, and was the guarantor.

\section{Funding/Support}

This article was a part of $\mathrm{PhD}$ thesis supported by Kerman University of Medical Sciences (International Campus).

\section{References}

1. World health Organization. Challenge of Adolescent Sexual Develop. Geneva; WHO. 2002.

2. Grunbaum JA, Kann L, Kinchen S, Ross J, Hawkins J, Lowry R. Youth Risk Behavior Surveillance. Surveillance Summaries. 2001.

3. Robertson EB, David SL, Rao SA. Preventing drug use among children and adolescents: A research-based guide for parents, educators, and community leaders. Diane Publishing; 2003.

4. Asonibare FB, Olowonireguaro OA. Family Cohesion and Level of Communication Between Parents and their Adolescent Children. Niger J Guid Couns . 2007;11(1):52-9.

5. Ensley MD, Pearson AW. An exploratory comparison of the behavioral dynamics of top management teams in family and nonfamily new ventures: Cohesion, conflict, potency, and consensus. Entrep Theory Pract. 2005;29(3):267-84.

6. Bomar PJ. Promoting Health in Families: Applying Family Research and Theory to nursing Practice. 3th ed: Elsevier Health Sciences; 2004. p. 96.

7. Beyers JM, Toumbourou JW, Catalano RF, Arthur MW, Hawkins JD. A cross-national comparison of risk and protective factors for adolescent substance use: the United States and Australia. J Adolesc Health. 2004;35(1):3-16.

8. De Micheli D, Formigoni ML. Drug use by Brazilian students: associations with family, psychosocial, health, demographic and behavioral characteristics. Addiction. 2004;99(5):570-8.

9. Steptoe A, Wardle J. Health-related behavior prevalence and links with disease. In: Kaptein A, Weinmen J editors. Health Psychology. BPS Blackwell; 2004.

10. Baban A, Craciun C. Changing health-risk behaviors: A review of theory and evidence-based interventions in health psychology. $J$ Cogn Behav Psychother. 2007;7(1):45-67.

11. Tu X, Lou C, Gao E, Li N. The Relationship Between Sexual Behavior and Nonsexual Risk Behaviors Among Unmarried Youth in Three Asian Cities. J Adolesc Health. 2012;50(3):75-82.

12. Ahmadi K. [Cultural, social and educational vulnerability in adolescents and youths].J Behav Sci. 2010;4(3):241-8.

13. Mancini JA, Huebner AJ. Adolescent risk behavior patterns: Effects of structured time-use, interpersonal connections, selfsystem characteristics, and socio-demographic influences. Child Adolesc Social Work J. 2004;21(6):647-68.

14. Mohammad Pourasl A, Fakhari A, Rostami F, Pourafkari NA [Cigarette smoking among Iranian adolescents]. Iran J Psychiatry Behav Sci. 2007;1(1):303-35.

15. Mohammad Pourasl A, Fakhari A, Rostami F, Vahidi R, Dastgiri S. [Epidemiology survey of the illicit drugs andalcohol use in sophomore male students of Tabriz's high schools].J Ardabil Univ Med Sci. 2007;7(4):400-8.

16. Golchin M. [Intrest in Aggression in adolescent and the role of the family].J Qazvin Univ Med Sci. 2002;21:35-40.

17. Golchin M. [Relationship Between Family Function and Some of Psychological Characters In Adolescents]. J Res Med Sci. 2002;6(4):297-9.

18. Olds RS, Thombs DL. The relationship of adolescent perceptions of peer norms and parent involvement to cigarette and alcohol use.J Sch Health. 2001;71(6):223-8.

19. U.S. Department of Health and Human Services Substance Abuse and Mental Health Services Administration. Communities that care youth survey normative database. In: Center for Substance Abuse Prevention, editor. Rockville; 2007.

20. Hawkins JD, Catalano RF, Miller JY. Risk and protective factors for alcohol and other drug problems in adolescence and early adulthood: implications for substance abuse prevention. Psychol Bull. 1992;112(1):64-105.

21. Terwee CB, Bot SD, de Boer MR, van der Windt DA, Knol DL, Dekker J, et al. Quality criteria were proposed for measurement properties of health status questionnaires. J Clin Epidemiol. 2007;60(1):34-42.

22. Mancini JA, Huebner AJ. Adolescent risk behavior patterns: Effects of structured time-use, interpersonal connections, selfsystem characteristics, and socio-demographic influences. Child Adolesc Soc Work J. 2004;21(6):647-68.

23. Huebner AJ, Howell LW. Examining the relationship between adolescent sexual risk-taking and perceptions of monitoring, communication, and parenting styles. J Adolesc Health. 2003; 33(2):71-8.

24. Kapungu CT, Holmbeck GN, Paikoff RL. Longitudinal association between parenting practices and early sexual risk behaviors among urban African American adolescents: The moderating role of gender.J Youth Adolesc. 2006;35(5):783-94. 


\section{Haghdoost A et al.}

25. McMahon TJ, Winkel JD, Suchman NE, Rounsaville BJ. Drug-abusing fathers: patterns of pair bonding, reproduction, and paternal involvement. J Subst Abuse Treat. 2007;33(3):295-302.

26. Farrington DP. Childhood aggression and adult violence: Early precursors and later life outcomes. The development and treatment of childhood aggression. 1991:5-29.

27. Hawkins JD, Catalano RF, Brewer DD. Preventing Serious,Violent and Chronic Juvenile Offending Effective Strategies in Childhood,Adolescence and the Community. In: Howell JC, Krisberg B, Hawkins JD, Wilson JJ editors. Serious, Violent and Chronic Juvenile Offenders. London: SAGE Publications; 1995.

28. Wisner B. At risk: natural hazards, people's vulnerability and disasters. Psychology Press; 2004.

29. Smith CA, Elwyn LJ, Ireland TO, Thornberry TP. Impact of adolescent exposure to intimate partner violence on substance use in early adulthood.J Stud Alcohol Drugs. 2010;71(2):219-30.

30. Kernic MA, Wolf ME, Holt VL, McKnight B, Huebner CE, Rivara FP. Behavioral problems among children whose mothers are abused by an intimate partner. Child Abuse Negl. 2003;27(11):1231-46.

31. Stewart A, Livingston M, Dennison S. Transitions and turning points: examining the links between child maltreatment and juvenile offending. Child Abuse Negl. 2008;32(1):51-66.

32. Sullivan ML. Illegal Leisure: The Normalization of Adolescent Recreational Drug Use/Understanding and Responding to Drug Use: The Role of Qualitative Research. Contemp Drug Probl. 2001;28(1):179-92.

33. Tucker JS, Ellickson PL, Collins RL, Klein DJ. Are drug experimenters better adjusted than abstainers and users?: a longitudinal study of adolescent marijuana use. $J$ Adolesc Health. 2006;39(4):488-94.

34. Panaghi L, Mohammadi S, Poshtmashhadi M, Zadehmohammadi A, Ahmadabadi Z. [High Risk Behaviors among Iranian Adolescents: Evaluating the Proportion of Family Factors]. Iran J Clin Psychol. 2012;1(1):38-47.

35. Aughinbaugh A, Gittleman M. Maternal employment and adolescent risky behavior. J Health Econ. 2004;23(4):815-38. 